\title{
DIFFERENTIABILITY OF CONTINUOUS FUNCTIONS IN TERMS OF HAAR-SMALLNESS
}

\author{
ADAM KWELA AND WOJCIECH ALEKSANDER WOEOSZYN
}

\begin{abstract}
One of the classical results concerning differentiability of continuous functions states that the set $\mathcal{S D}$ of somewhere differentiable functions (i.e., functions which are differentiable at some point) is Haar-null in the space $C[0,1]$. By a recent result of Banakh et al., a set is Haar-null provided that there is a Borel hull $B \supseteq A$ and a continuous map $f:\{0,1\}^{\mathbb{N}} \rightarrow C[0,1]$ such that $f^{-1}[B+h]$ is Lebesgue's null for all $h \in C[0,1]$.

We prove that $\mathcal{S D}$ is not Haar-countable (i.e., does not satisfy the above property with "Lebesgue's null" replaced by "countable", or, equivalently, for each copy $C$ of $\{0,1\}^{\mathbb{N}}$ there is an $h \in C[0,1]$ such that $\mathcal{S D} \cap(C+h)$ is uncountable.

Moreover, we use the above notions in further studies of differentiability of continuous functions. Namely, we consider functions differentiable on a set of positive Lebesgue's measure and functions differentiable almost everywhere with respect to Lebesgue's measure. Furthermore, we study multidimensional case, i.e., differentiability of continuous functions defined on $[0,1]^{k}$. Finally, we pose an open question concerning Takagi's function.
\end{abstract}

\section{INTRODUCTION}

We follow the standard topological notation and terminology. By $|X|$ we denote the cardinality of a set $X$.

For a function $f \in C[0,1]$, by $D(f)$ we denote the set of all points $x \in[0,1]$ at which $f$ is differentiable. There are examples of continuous functions such that $D(f)=\varnothing$. One of the first and simplest examples is the famous Takagi's function $T: \mathbb{R} \rightarrow \mathbb{R}$ given by $T(x)=\sum_{i=0}^{\infty} \frac{1}{2^{i}} \operatorname{dist}\left(2^{i} x, \mathbb{Z}\right)$ (see [1, 2], or [15]). The size of the set of somewhere differentiable functions, i.e., functions $f$ such that $D(f) \neq \varnothing$, is a classical object of studies since Banach's result stating that this set is meager in $C[0,1]$ (cf. 44). One of the well-known results in this subject is Hunt's theorem stating that the aforementioned set is Haar-null in the space $C[0,1]$ (see [12]). This notion was first introduced in 8 by Christensen. He called a subset $A$ of an abelian Polish group $X$ Haar-null provided that there is a Borel hull $B \supseteq A$ and a Borel $\sigma$-additive probability measure $\lambda$ on $X$ such that $\lambda(B+x)=0$ for all $x \in X$ (actually, in the original paper Christensen introduced this notion using universally measurable hulls instead of Borel hulls, however in many papers, e.g. [5] or [9], Haar-null sets are defined in the same way as above). A big advantage of this concept is that in a locally compact group it is equivalent to the notion of Haar measure zero sets and at the same time it can be used in a significantly larger class of groups. In [13. Hunt, Sauer and Yorke, unaware of Christensen's paper,

2010 Mathematics Subject Classification. Primary: 26A27; secondary: 03E15, 26B05, 54H11.

Key words and phrases. Nowhere differentiable functions, Continuous functions, Haar-small sets, Haar-null sets, Haar-meager sets. 
reintroduced the notion of Haar-null sets in the context of dynamical systems (in their paper Haar-null sets are called shy sets, and their complements are called prevalent sets).

Actually, since the set of somewhere differentiable functions is not Borel, Hunt had to show something more: the set of somewhere Lipschitz functions is Haarnull in $C[0,1]$ (a function $f \in C[0,1]$ is somewhere Lipschitz whenever there is an $x \in[0,1]$ and an $M \in \mathbb{N}$ such that $|f(x)-f(y)| \leq M|x-y|$ for each $y \in[0,1]$; observe that each somewhere differentiable function is somewhere Lipschitz).

In this paper, we are interested in the following notions of smallness. A subset $A$ of an abelian Polish group $X$ is:

- Haar-countable if there is a Borel hull $B \supseteq A$ and a copy $C$ of $\{0,1\}^{\mathbb{N}}$ such that $(C+x) \cap B$ is countable for all $x \in X$;

- Haar-finite if there is a Borel hull $B \supseteq A$ and a copy $C$ of $\{0,1\}^{\mathbb{N}}$ such that $(C+x) \cap B$ is finite for all $x \in X$;

- Haar- $n$, for $n \in \mathbb{N}$, if there is a Borel hull $B \supseteq A$ and a copy $C$ of $\{0,1\}^{\mathbb{N}}$ such that $|(C+x) \cap B| \leq n$ for all $x \in X$.

Clearly,

$$
\text { Haar- } n \Longrightarrow \text { Haar- }(n+1) \Longrightarrow \text { Haar-finite } \Longrightarrow \text { Haar-countable }
$$

for any $n \in \mathbb{N}$.

The choice of names in the above is due to Banakh et al., who recently unified the notions of Haar-null sets and Haar-meager sets (defined by Darji in [9]) in [5] by introducing the concept of Haar-small sets. A collection of subsets of a set $X$ is called a semi-ideal whenever it is closed under taking subsets. Following [5, Definition 11.1], for a semi-ideal $\mathcal{I}$ on the Cantor cube $\{0,1\}^{\mathbb{N}}$, we say that a subset $A$ of an abelian Polish group $X$ is Haar- $\mathcal{I}$ if there is a Borel hull $B \supseteq A$ and a continuous map $f:\{0,1\}^{\mathbb{N}} \rightarrow X$ such that $f^{-1}[B+x] \in \mathcal{I}$ for all $x \in X$. It turns out that if $\mathcal{I}$ is the $\sigma$-ideal $\mathcal{N}$ of subsets of $\{0,1\}^{\mathbb{N}}$ of Haar measure zero, then we obtain Haar-null sets (cf. [5, Theorem 11.5]). The same holds for the $\sigma$-ideal $\mathcal{M}$ of meager sets and Haar-meager sets (cf. [5, Theorem 11.5]), the $\sigma$-ideal of countable sets and Haar-countable sets, the ideal of finite sets and Haar-finite sets and the semi-ideal of sets of cardinality at most $n$ and Haar- $n$ sets (cf. 114, Proposition $1.2])$.

Obviously, the collection of Haar- $\mathcal{I}$ subsets of an abelian Polish group is a semiideal. Observe that $\mathcal{I} \subseteq \mathcal{J}$ implies that each Haar- $\mathcal{I}$ set is Haar- $\mathcal{J}$. Thus, Haar-null sets and Haar-meager sets only allow us to say that some properties are rare (and we cannot put them on a scale and compare with other rare properties), whereas Haar- $\mathcal{I}$ sets allow us to develop a whole hierarchy of small sets.

Haar-countable, Haar-finite, and Haar- $n$ sets were profoundly studied by the first author in 14. There are compact examples showing that none of the above implications can be reversed in $C(K)$ where $K$ is compact metrizable. Moreover, it is known that neither Haar-finite sets nor Haar- $n$ sets form an ideal (see [14, Corollary 5.2 and Theorem 6.1]). Zakrzewski considered Haar-small sets in [17] under the name of perfectly $\kappa$-small sets. A particular case of Haar-1 sets was investigated by Balcerzak in [3]. He introduced the so-called property (D) of a $\sigma$ ideal $\mathcal{I}$, which says that there is a Borel Haar-1 set not belonging to $\mathcal{I}$. Moreover, Banakh, Lyaskovska, and Repovš considered packing index of a set in [6]. Packing index is closely connected to Haar-1 sets (namely, a Borel set is Haar-1 if and only 
if its packing index is uncountable). In turn, Haar-countable sets were studied by Darji and Keleti in [10] and by Elekes and Steprāns in [11].

\section{Nowhere Differentiable FunCtions}

Hunt proved in [12 that the set $\mathcal{S D}$ of somewhere differentiable functions is Haar-null in $C[0,1]$. Actually, a closer look at his proof gives us something more. Denote by $\mathcal{E}(\mathcal{E}(\mathbb{R}))$ the $\sigma$-ideal generated by compact Haar null subsets of $\{0,1\}^{\mathbb{N}}$ $(\mathbb{R}$, respectively) and recall that $\mathcal{E}$ is a proper subfamily of $\mathcal{N} \cap \mathcal{M}$ (see [7, Lemma 2.6.1]). Hunt's proof shows that there is a continuous function $\varphi: \mathbb{R}^{2} \rightarrow C[0,1]$ such that $\varphi^{-1}[f+\mathcal{S D}] \in \mathcal{E}(\mathbb{R})$ for all $f \in C[0,1]$. If $\psi:\{0,1\}^{\mathbb{N}} \rightarrow[0,1]^{2}$ denotes the map given by $\psi\left(\left(x_{i}\right)\right)=\left(\sum_{i \in \mathbb{N}} \frac{x_{2 i-1}}{2^{i}}, \sum_{i \in \mathbb{N}} \frac{x_{2 i}}{2^{i}}\right)$ then $\varphi^{-1}[A] \in \mathcal{E}$ for all $A \in \mathcal{E}(\mathbb{R})$. Thus, $\psi^{-1}\left[\varphi^{-1}[f+\mathcal{S D}]\right] \in \mathcal{E}$ for all $f \in C[0,1]$, i.e., we have shown the following:

Theorem 2.1 (Hunt, [12]). The set $\mathcal{S D}$ is Haar-E in $C[0,1]$.

In this Section we show that $\mathcal{S D}$ is not Haar-countable in $C[0,1]$.

Theorem 2.2. The set of somewhere differentiable functions is not Haar-countable in $C[0,1]$.

Proof. Denote by $C$ the ternary Cantor set (which is homeomorphic to $\{0,1\}^{\mathbb{N}}$ ). Let $\varphi: C \rightarrow C[0,1]$ be continuous. We need to find a homeomorphic copy $C^{\prime}$ of $C$ and a continuous function $g:[0,1] \rightarrow \mathbb{R}$ such that $\varphi(c)-g \in \mathcal{S D}$ for all $c \in C^{\prime}$.

Define $D_{1}=\{0,1\}$ and $D_{n}=\left(\left\{\frac{i}{3^{n-1}}: i \in \mathbb{N}\right\} \cap C\right)$ for all $n \in \mathbb{N}, n \neq 1$. Let $E_{1}=D_{1}$ and $E_{n}=D_{n} \backslash D_{n-1}$, for $n \in \mathbb{N}, n>1$. Observe that $D=\bigcup_{n \in \omega} D_{n}$ is the set of all points from $C$ with finite ternary expansion. For each $n \in \mathbb{N}$ and $d \in E_{n}$, let:

$$
U_{d}=[0,1] \cap\left[d-\frac{\frac{1}{3^{n-1}}+\frac{1}{3^{n+1}}}{2}, d+\frac{\frac{1}{3^{n-1}}+\frac{1}{3^{n+1}}}{2}\right] .
$$

Note that $U_{d} \cap D_{n}=\{d\}$, for every $d \in D_{n}$.

First, we need to shrink $C$. Namely, we want to have a homeomorphic copy $C^{\prime} \subseteq C$ of $C$ such that:

- $\left\|f_{c}-f_{c^{\prime}}\right\| \leq\left(c-c^{\prime}\right)^{2}$, for all $c, c^{\prime} \in C$;

- $\left\|f_{d}-f_{d^{\prime}}\right\|<\inf _{x \in U_{d} \cap U_{d^{\prime}}}\left((d-x)^{2}+\left(d^{\prime}-x\right)^{2}\right)$, for all distinct $d, d^{\prime} \in D$ with $U_{d} \cap U_{d^{\prime}} \neq \varnothing$;

- $\left\|f_{d}-f_{d^{\prime}}\right\|<\frac{1}{5}\left(\operatorname{dist}\left(d, U_{d^{\prime}}\right)\right)^{2}=\frac{1}{5}\left(\operatorname{dist}\left(d^{\prime}, U_{d}\right)\right)^{2}$, whenever $d, d^{\prime} \in E_{n}$ for some $n, d \neq d^{\prime}$ and $U_{d} \cap U_{d^{\prime}} \neq \varnothing$;

- $\left\|f_{d}-f_{d^{\prime}}\right\|<\frac{1}{5}\left(\operatorname{dist}\left(d, U_{d^{\prime}}\right)\right)^{2}$, whenever $d^{\prime} \in E_{n}$ and $d \in D_{n} \backslash E_{n}$ for some $n$ and $U_{d} \cap U_{d^{\prime}} \neq \varnothing$

where $f_{x}=\varphi(\psi(x))$ and $\psi: C \rightarrow C^{\prime}$ is the aforementioned homeomorphism.

The construction of $C^{\prime}$ is rather standard. Nevertheless, we provide a short sketch of it.

Let $e: C^{2} \rightarrow \mathbb{R}$ be a continuous function such that $\left\|f_{c}-f_{c^{\prime}}\right\| \leq e\left(c, c^{\prime}\right)$ guarantees all of the above conditions and $e\left(c, c^{\prime}\right)>0$ for all $c, c^{\prime} \in C$ with $c \neq c^{\prime}$ (which clearly exists). For a finite sequence $s=\left(s_{1}, \ldots, s_{n}\right) \in\{0,1\}^{n}$, let $\bar{s}=\sum_{i=1}^{n} \frac{2 s_{i}}{3^{i}}$. In the first inductive step pick any basic clopen set $W_{\varnothing} \subseteq C$ such that:

$$
\operatorname{diam}\left(\varphi\left[W_{\varnothing}\right]\right)<\inf \left\{e\left(c, c^{\prime}\right): c \in C \cap\left[0, \frac{1}{3}\right] \wedge c^{\prime} \in C \cap\left[\frac{2}{3}, 1\right]\right\} .
$$


If $W_{s}$ are already defined for all $s \in\{0,1\}^{n}$, for each $s=\left(s_{1}, \ldots, s_{n}\right) \in\{0,1\}^{n}$ find two disjoint basic clopen sets $W_{s \frown(0)}, W_{s \frown(1)} \subseteq W_{s}$ such that $\operatorname{diam}\left(\varphi\left[W_{s \frown(i)}\right]\right)<$ $\frac{1}{2^{n+1}}$ and:

$$
\begin{gathered}
\operatorname{diam}\left(\varphi\left[W_{s \frown(i)}\right]\right)<\inf \left\{e\left(c, c^{\prime}\right): c \in C \cap\left[\overline{s \frown(i, 0)}, \overline{s \frown(i, 0)}+\frac{1}{3^{n+1}}\right] \wedge\right. \\
\left.\wedge c^{\prime} \in C \cap\left[\overline{s \frown(i, 1)}, \overline{s \frown(i, 1)}+\frac{1}{3^{n+1}}\right]\right\},
\end{gathered}
$$

for $i=0,1$. It is easy to check that $C^{\prime}=\bigcup_{x \in\{0,1\}^{\mathbb{N}}} \bigcap_{n \in \mathbb{N}} W_{x \mid\{1, \ldots, n\}}$ is the required set.

Now, we want to construct a $g \in C[0,1]$ such that $f_{c}-g$ has a derivative at $c$ equal to 0 , for all $c \in C$. Inductively, we will define a sequence of continuous functions $\left(g_{n}\right) \subseteq C[0,1]$ such that:

$$
\begin{aligned}
& \forall \forall_{d \in E_{n}} \forall_{x \in U_{d}}\left|f_{d}(x)-\sum_{i=1}^{n} g_{i}(x)\right| \leq \frac{1}{5}(x-d)^{2} ; \\
& \forall_{d \in D_{n}} \forall_{x \in U_{d}}\left|f_{d}(x)-\sum_{i=1}^{n} g_{i}(x)\right| \leq(x-d)^{2} .
\end{aligned}
$$

At the end, we will put $g=\sum_{i=1}^{\infty} g_{n}$.

Start the construction with $g_{1} \in C[0,1]$ such that:

- $g_{1}=f_{0}$ on $U_{0} \backslash U_{1}$

- $g_{1}=f_{1}$ on $U_{1} \backslash U_{0}$;

- $g_{1}(x)$ is between $f_{0}(x)$ and $f_{1}(x)$ for all $x \in U_{0} \cap U_{1}$.

Note that we have $\left|f_{0}(x)-g_{1}(x)\right|=0 \leq \frac{1}{5}(x-0)^{2}$ for all $x \in U_{1} \backslash U_{0}$ and $\left|f_{0}(x)-g_{1}(x)\right| \leq\left\|f_{0}-f_{1}\right\|<\frac{1}{5}\left(\inf U_{1}-0\right)^{2} \leq \frac{1}{5}(x-0)^{2}$ for all $x \in U_{0} \cap U_{1}$. Similarly, $\left|f_{1}(x)-g_{1}(x)\right| \leq \frac{1}{5}(x-1)^{2}$ for all $x \in U_{1}$. Thus, $g_{1}$ is as needed.

Once all $g_{i}$ 's, for $i<n$, are defined, let $\tilde{g}_{n}: \bigcup_{d \in E_{n}} U_{d} \rightarrow \mathbb{R}$ be a continuous function such that:

- $\tilde{g}_{n}=f_{d}-\sum_{i=1}^{n-1} g_{i}$ on $U_{d} \backslash \bigcup_{d^{\prime} \in E_{n} \backslash\{d\}} U_{d^{\prime}}$ for each $d \in E_{n}$;

- if $d, d^{\prime} \in E_{n}$ and $U_{d} \cap U_{d^{\prime}} \neq \varnothing$, then $\tilde{g}_{n}(x)$ is between $f_{d}(x)-\sum_{i=1}^{n-1} g_{i}(x)$ and $f_{d^{\prime}}(x)-\sum_{i=1}^{n-1} g_{i}(x)$ for all $x \in U_{d} \cap U_{d^{\prime}}$.

Let $d \in E_{n}$ and notice that $\left|f_{d}(x)-\sum_{i=1}^{n-1} g_{i}(x)-\tilde{g}_{n}(x)\right|=0 \leq \frac{1}{5}(x-d)^{2}$ for all $x \in U_{d} \backslash \bigcup_{d^{\prime} \in E_{n} \backslash\{d\}} U_{d^{\prime}}$ and $\left|f_{d}(x)-\sum_{i=1}^{n-1} g_{i}(x)-\tilde{g}_{n}(x)\right| \leq\left\|f_{d}-f_{d^{\prime}}\right\|<$ $\frac{1}{5}\left(\operatorname{dist}\left(d, U_{d^{\prime}}\right)\right)^{2} \leq \frac{1}{5}(x-d)^{2}$ for all $x \in U_{d} \cap U_{d^{\prime}}$ whenever the latter intersection is non-empty. Moreover, if $d \in D_{n} \backslash E_{n}$ and $x \in U_{d} \cap U_{d^{\prime}}$ for some $d^{\prime} \in E_{n}$, then:

$$
\begin{gathered}
\left|d^{\prime}-x\right| \leq \frac{\frac{1}{3^{n-1}}+\frac{1}{3^{n+1}}}{2} \leq \frac{1}{3^{n-1}}-\frac{1}{3^{n+1}}= \\
2\left(\frac{1}{3^{n-1}}-\frac{\frac{1}{3^{n-1}}+\frac{1}{3^{n+1}}}{2}\right) \leq 2 \operatorname{dist}\left(d, U_{d^{\prime}}\right) \leq 2|d-x| .
\end{gathered}
$$

Therefore,

$$
\left|f_{d}(x)-\sum_{i=1}^{n-1} g_{i}(x)-\tilde{g}_{n}(x)\right| \leq\left\|f_{d}-f_{d^{\prime}}\right\|+\left|f_{d^{\prime}}(x)-\sum_{i=1}^{n-1} g_{i}(x)-\tilde{g}_{n}(x)\right| \leq
$$


DIFFERENTIABILITY OF CONTINUOUS FUNCTIONS IN TERMS OF HAAR-SMALLNESS 5

$$
\leq \frac{1}{5}\left(\operatorname{dist}\left(d, U_{d^{\prime}}\right)\right)^{2}+\frac{1}{5}\left(d^{\prime}-x\right)^{2} \leq \frac{1}{5}(d-x)^{2}+\frac{4}{5}(d-x)^{2}=(d-x)^{2} .
$$

Hence, $\tilde{g}_{n}$ satisfies all the required conditions and it suffices to extend it to a continuous function $g_{n}$ defined on the whole interval $[0,1]$ such that:

$$
\left(f_{d}(x)-\sum_{i=1}^{n-1} g_{i}(x)-(d-x)^{2}\right) \leq g_{n}(x) \leq\left(f_{d}(x)-\sum_{i=1}^{n-1} g_{i}(x)+(d-x)^{2}\right),
$$

for all $d \in D_{n}$ such that $x \in U_{d}$. Note that such extension exists. Indeed, we need to check that whenever $x \in U_{d} \cap U_{d^{\prime}}$ for some $d, d^{\prime} \in D_{n} \backslash E_{n}$ then

$$
f_{d}(x)-\sum_{i=1}^{n-1} g_{i}(x)-(d-x)^{2} \leq f_{d^{\prime}}(x)-\sum_{i=1}^{n-1} g_{i}(x)+\left(d^{\prime}-x\right)^{2} .
$$

The above leads to $\left|f_{d}(x)-f_{d^{\prime}}(x)\right| \leq(d-x)^{2}+\left(d^{\prime}-x\right)^{2}$ which is satisfied as $\left\|f_{d}-f_{d^{\prime}}\right\|<\inf _{x \in U_{d} \cap U_{d^{\prime}}}\left((d-x)^{2}+\left(d^{\prime}-x\right)^{2}\right)$, for all distinct $d, d^{\prime} \in D$ with $U_{d} \cap$ $U_{d^{\prime}} \neq \varnothing$.

Once the construction is completed, define $g=\sum_{n=1}^{\infty} g_{n}$. For each $d \in D$ and $x \in U_{d}$, we have $\left|f_{d}(x)-g(x)\right| \leq(x-d)^{2}$. Thus, $f_{d}-g$ has a derivative at $d$ equal to 0 . Now, we want to show that $f_{c}-g$ has a derivative at $c$ equal to 0 for each $c \in C$.

Fix $c \in C \backslash D$ and $h \in \mathbb{R}$ such that $c+h \in[0,1]$. Assume that $c+h \in U_{d}$ for some $d \in D$ and $|h| \geq \frac{1}{4}|c-d|$. Observe that:

$$
\begin{gathered}
\left|\frac{f_{c}(c+h)-g(c+h)-f_{c}(c)+g(c)}{h}\right| \leq \\
\leq\left|\frac{f_{c}(c+h)-f_{d}(c+h)}{h}\right|+\left|\frac{f_{d}(c+h)-g(c+h)-f_{d}(c)+g(c)}{h}\right|+\left|\frac{f_{d}(c)-f_{c}(c)}{h}\right| \leq \\
\leq 2 \frac{\left\|f_{c}-f_{d}\right\|}{|h|}+\left|\frac{f_{d}(c+h)-g(c+h)-f_{d}(c)+g(c)}{h}\right| .
\end{gathered}
$$

As $\left\|f_{c}-f_{d}\right\| \leq(c-d)^{2}$ and $|h| \geq \frac{1}{4}|c-d|$, we get that $\frac{\left\|f_{c}-f_{d}\right\|}{|h|} \leq 4|c-d|$. What is more, if $d^{\prime} \in D$ is such that $c \in U_{d^{\prime}}$ then

$\left|f_{d}(c)-g(c)\right| \leq\left|f_{d}(c)-f_{d^{\prime}}(c)\right|+\left|f_{d^{\prime}}(c)-g(c)\right| \leq\left(d-d^{\prime}\right)^{2}+\left(d^{\prime}-c\right)^{2} \leq(d-c)^{2}$.

Using $|h| \geq \frac{1}{4}|c-d|$ once again, we have:

$$
\begin{gathered}
\left|\frac{f_{d}(c+h)-g(c+h)-f_{d}(c)+g(c)}{h}\right| \leq \frac{(c+h-d)^{2}+(c-d)^{2}}{|h|}= \\
=\frac{2(c-d)^{2}+2 h(c-d)+h^{2}}{|h|} \leq 8|c-d|+2|c-d|+|h| .
\end{gathered}
$$

Thus, $\frac{f_{c}(c+h)-g(c+h)-f_{c}(c)+g(c)}{h}$ tends to 0 as $h \rightarrow 0$ and $|c-d| \rightarrow 0$. Therefore, to finish the proof, it suffices to show that for each $c \in C$ there are sequences $\left(d_{n}\right) \subseteq D \cap(c, 1]$ and $\left(d_{n}^{\prime}\right) \subseteq D \cap[0, c)$ converging to $c$ and such that $(c, 1] \subseteq$ $\bigcup_{n \in \mathbb{N}} U_{d_{n}} \cap\left[c+\frac{1}{4}\left(d_{n}-c\right), 1\right]$ and $[0, c) \subseteq \bigcup_{n \in \mathbb{N}} U_{d_{n}^{\prime}} \cap\left[0, c-\frac{1}{4}\left(c-d_{n}^{\prime}\right)\right]$.

We will construct the sequence $\left(d_{n}\right)$. Construction of $\left(d_{n}^{\prime}\right)$ is similar. Let $\left(c_{n}\right) \in\{0,2\}^{\mathbb{N}}$ be the ternary expansion of $c \in C$ and $\left(i_{n}\right) \subseteq \mathbb{N}$ be the increasing enumeration of the set $\left\{n \in \mathbb{N}: c_{n}=0\right\}$. For all $k \in \mathbb{N}$, define $d_{1}=1$, $d_{2 k}=1-\sum_{j=1}^{k-1} \frac{2}{3^{i} j}-\frac{1}{3^{i} k}$, and $d_{2 k+1}=1-\sum_{j=1}^{k-1} \frac{2}{3^{i} j}-\frac{2}{3^{i} k}$. Now, we show that $\left(d_{n}\right)$ is as required. 
We need to show that $\sup U_{d_{n+1}}>c+\frac{1}{4}\left(d_{n}-c\right)$ for all $n \in \mathbb{N}$. Clearly, $\sup U_{d_{2 k}} \geq$ $d_{2 k}+\frac{\frac{1}{3^{i_{k}}+\frac{1}{3^{i} k+2}}}{2}$ and $\sup U_{d_{2 k+1}} \geq d_{2 k+1}+\frac{\frac{1}{3^{i} k}+\frac{1}{3^{2} k+2}}{2}$, i.e., it suffices to show:

$$
\begin{aligned}
& d_{2 k}+\frac{\frac{1}{3^{i_{k}}}+\frac{1}{3^{i_{k}+2}}}{2}>c+\frac{1}{4}\left(d_{2 k-1}-c\right) ; \\
& d_{2 k+1}+\frac{\frac{1}{3^{i} k}+\frac{1}{3^{i_{k}+2}}}{2}>c+\frac{1}{4}\left(d_{2 k}-c\right) .
\end{aligned}
$$

As $d_{2 k}=c+\left(d_{2 k}-c\right)$ and $d_{2 k+1}=c+\left(d_{2 k+1}-c\right)$, this is equivalent to:

$$
\begin{aligned}
& d_{2 k}-c+\frac{\frac{1}{2}+\frac{1}{18}}{3^{i_{k}}}>\frac{1}{4}\left(d_{2 k-1}-c\right) ; \\
& d_{2 k+1}-c+\frac{\frac{1}{2}+\frac{1}{18}}{3^{i_{k}}}>\frac{1}{4}\left(d_{2 k}-c\right) .
\end{aligned}
$$

Multiplying both sides by 4 and using $d_{2 k-1}=d_{2 k}+\frac{1}{3^{i k}}$ and $d_{2 k}=d_{2 k+1}+\frac{1}{3^{i k}}$ we get:

$$
\begin{gathered}
4\left(d_{2 k}-c\right)+\frac{2+\frac{2}{9}}{3^{i_{k}}}>d_{2 k}-c+\frac{1}{3^{i_{k}}} \\
4\left(d_{2 k+1}-c\right)+\frac{2+\frac{2}{9}}{3^{i_{k}}}>d_{2 k+1}-c+\frac{1}{3^{i_{k}}} .
\end{gathered}
$$

Thus,

$$
\begin{gathered}
3\left(d_{2 k}-c\right)>\frac{1-2-\frac{2}{9}}{3^{i_{k}}} ; \\
3\left(d_{2 k+1}-c\right)>\frac{1-2-\frac{2}{9}}{3^{i_{k}}} ;
\end{gathered}
$$

which is true since $d_{2 k}>c$ and $d_{2 k+1}>c$. This finishes the entire proof.

By the above, the set of functions differentiable at some point is not Haarcountable. However, what about functions differentiable at more than one point? As for a given $\sigma$-ideal $\mathcal{I}$ on $[0,1]$ the set $\{f \in C[0,1]: \varnothing \neq D(f) \in \mathcal{I}\}$ is contained in the set of somewhere differentiable functions, this question is natural. The following slight strengthening of Theorem 2.2 gives only a partial answer to this problem.

Corollary 2.3. Let $\mathcal{I}$ be a $\sigma$-ideal on $[0,1]$ containing some perfect set. The set of functions $f \in C[0,1]$ such that $D(f)$ is a nonempty set which belongs to $\mathcal{I}$ is not Haar-countable.

Proof. First, assume that the ternary Cantor set $C$ belongs to $\mathcal{I}$. We need to make two modifications of the proof of Theorem 2.2,

Since, by Hunt's result, the set of somewhere Lipschitz functions is Borel and Haar-null (see [12), the set $\mathcal{N} \mathcal{L}$ of nowhere Lipschitz functions cannot be Haarnull. Thus, for $\varphi$ from the proof of Theorem 2.2, there is a $z \in C[0,1]$ such that $\varphi^{-1}[\mathcal{N} \mathcal{L}-z]$ is not Lebesgue's null. In particular, this is a Borel uncountable subset of $C$. Hence, it must contain a homeomorphic copy $P$ of $C$. Then, $\varphi(c)+z$ is nowhere differentiable for each $c \in P$. Thus, by performing the construction of $C^{\prime}$ inside $P$ and defining $f_{x}=\varphi(\psi(x))+z$ where $\psi$ is a homeomorphism from $C$ to $C^{\prime}$, we may assume that $f_{c}$ is nowhere differentiable for each $c \in C$. Moreover, this changes do not affect the rest of the proof. If we find $g \in C[0,1]$ such that $f_{c}-g$ is somewhere differentiable for all $c \in C$, then $\varphi(c)+(z-g)$ is somewhere differentiable for uncountably many $c \in C$ (namely, for all $c \in C^{\prime}$ ). 
Now, we move to the second modification. We can ensure that $g$ is differentiable outside $C$. Indeed, for each connected component $I$ of $[0,1] \backslash C$ fix a sequence of closed (possibly empty) intervals $\left(I_{n}\right)$ such that $\bigcup_{n} I_{n}=I, I_{n} \subseteq I_{n+1}$ and $I_{n} \cap \bigcup_{d \in E_{n}} U_{d}=\varnothing$ for all $n$ (which is possible as $\bigcup_{n} E_{n} \subseteq C$ and $\sup _{d \in E_{n}} \operatorname{diam}\left(U_{d}\right)$ tends to 0 as $n \rightarrow+\infty$ ). Now it suffices to require, additionally, in the inductive construction of $\left(g_{n}\right)$, that $\sum_{i=1}^{n} g_{i}$ is differentiable on $I_{n}$ and $g_{n} \uparrow I_{n-1}=0$ (if $n>1$ ). This can be done as for $x \in I_{n}$ the only requirement imposed on $g_{n}$ resulting from the proof of Theorem 2.2 is:

$$
\sup _{d \in D_{n}, x \in U_{d}}\left(f_{d}(x)-(d-x)^{2}\right) \leq \sum_{i=1}^{n} g_{i}(x) \leq \inf _{d \in D_{n}, x \in U_{d}}\left(f_{d}(x)+(d-x)^{2}\right) .
$$

Thus, there is no problem with the request $g_{n}\left\lceil I_{n-1}=0\right.$ as $\left(\bigcup_{d \in D_{n}} U_{d}\right) \cap I_{n-1} \subseteq$ $\left(\bigcup_{d \in D_{n}} U_{d}\right) \cap I_{n} \subseteq \bigcup_{d \in D_{n-1}} U_{d}$ and in the $n$th induction step we already have:

$$
\sup _{d \in D_{n-1}, x \in U_{d}}\left(f_{d}(x)-(d-x)^{2}\right) \leq \sum_{i=1}^{n-1} g_{i}(x) \leq \inf _{d \in D_{n-1}, x \in U_{d}}\left(f_{d}(x)+(d-x)^{2}\right) .
$$

What is more, $\sum_{i=1}^{n-1} g_{i}(x)$ is differentiable on $I_{n-1}$, hence so is $\sum_{i=1}^{n} g_{i}(x)$. Since

$$
\sup _{d \in D_{n}, x \in U_{d}}\left(f_{d}(x)-(d-x)^{2}\right)<\inf _{d \in D_{n}, x \in U_{d}}\left(f_{d}(x)+(d-x)^{2}\right)
$$

(by the fact that $\left\|f_{d}-f_{d^{\prime}}\right\|<\inf _{x \in U_{d} \cap U_{d^{\prime}}}\left((d-x)^{2}+\left(d^{\prime}-x\right)^{2}\right)$, for all distinct $d, d^{\prime} \in D$ with $\left.U_{d} \cap U_{d^{\prime}} \neq \varnothing\right)$, there is some freedom in the choice of $g_{n}$ and we can ensure that $\sum_{i=1}^{n} g_{i}$ is differentiable also on $I_{n} \backslash I_{n-1}$.

After these modifications, as $g$ is differentiable at each point $x \in[0,1] \backslash C$ while $f_{c}$ is not, $f_{c}-g$ is not differentiable at each point of $[0,1] \backslash C$ and we get that $D\left(f_{c}-g\right) \subseteq C$ for all $c \in C$.

The case where $C \notin \mathcal{I}$ requires one additional modification. Since every perfect set contains a homeomorphic copy of the ternary Cantor set $C$, we simply need to find such a copy $R$ that belongs to $\mathcal{I}$. Then, we can replace $C$ with $R$, modify sets $D_{n}$ and $U_{d}$, and perform similar reasoning as in the proof of Theorem 2.2

It is known that $D(f)$ is Borel (of type $\mathrm{G}_{\delta \sigma}$ ) for each $f \in C[0,1]$ (see [16]). Thus, we can consider Lebesgue's measure of the set $D(f)$. Since there are perfect sets of Lebesgue's measure zero, the following is immediate.

Corollary 2.4. The set of functions $f \in C[0,1]$ such that $D(f)$ is a nonempty set of Lebesgue's measure zero is not Haar-countable.

As the $\sigma$-ideal of countable sets does not contain any perfect set, the following question arises.

Problem 2.5. Is the set of functions $f \in C[0,1]$ such that $D(f)$ is countable but non-empty Haar-countable in $C[0,1]$ ?

\section{Differentiability and Lebesgue's measure}

In this Section, we examine functions differentiable on a set of positive Lebesgue's measure. 
We will need the following notation. By the symbol $\lambda$ we will denote the Lebesgue's measure on $[0,1]$. Moreover, for a function $f \in C[0,1]$ and $M \in \mathbb{N}$, define

$$
L_{M}(f)=\left\{x \in[0,1]: \forall_{y \in[0,1]}|f(x)-f(y)| \leq M|x-y|\right\} .
$$

Then, $f$ is somewhere Lipschitz if and only if the set $L(f)=\bigcup_{M \in \mathbb{N}} L_{M}(f)$ is non-empty.

The next two rather folklore lemmas will be useful in our further considerations.

Lemma 3.1. For any $f \in C[0,1]$ and $M \in \mathbb{N}$, the set $L_{M}(f)$ is closed.

Proof. We will show that $[0,1] \backslash L_{M}(f)$ is open. Fix any $x \in[0,1] \backslash L_{M}(f)$. Then, there is $y \in[0,1]$ such that $|f(x)-f(y)|>M|x-y|$. Find $\alpha>0$ such that $|f(x)-f(y)|>M(|x-y|+\alpha)$. By continuity of $f$ at $x$, there is a $\delta>0$ such that

$$
|f(x)-f(z)|<|f(x)-f(y)|-M(|x-y|+\alpha)
$$

whenever $|x-z|<\delta$. Then, for each $z \in[0,1]$ such that $|x-z|<\min \{\delta, \alpha\}$, we have:

$$
|f(z)-f(y)| \geq|| f(x)-f(y)|-| f(x)-f(z)||>M(|x-y|+\alpha)>M|y-z| .
$$

Hence, $z \notin L_{M}(f)$.

This result implies that $L(f)$ is Borel (of type $\mathbf{F}_{\sigma}$ ). Thus, we can consider Lebesgue's measure of the sets $L_{M}(f)$ and $L(f)$.

Lemma 3.2. For each $M \in \mathbb{N}$ and $c \in(0,1]$, the set of functions $f \in C[0,1]$ such that $\lambda\left(L_{M}(f)\right) \geq c$ is closed in $C[0,1]$.

Proof. Fix a sequence $\left(f_{n}\right) \subseteq C[0,1]$ converging to some $f \in C[0,1]$ and such that $\lambda\left(L_{M}\left(f_{n}\right)\right) \geq c$ for each $n$. We need to show that $\lambda\left(L_{M}(f)\right) \geq c$. Suppose, to the contrary, that $\lambda\left(L_{M}(f)\right)<c$. Using regularity of Lebesgue's measure, find an open set $G \subseteq[0,1]$ such that $L_{M}(f) \subseteq G$ and $\lambda(G)<c$.

For each $n$, there is an $x_{n} \in L_{M}\left(f_{n}\right) \backslash G\left(\right.$ as $\left.\lambda(G)<\lambda\left(L_{M}\left(f_{n}\right)\right)\right)$. Since $[0,1]$ is compact, without loss of generality we may assume that $\left(x_{n}\right)$ converges to some $x \in[0,1]$. Observe that $x \notin L_{M}(f)$ as $G$ is an open hull of $L_{M}(f)$ and whole sequence $\left(x_{n}\right)$ is outside of $G$. However,

$$
\begin{gathered}
|f(x)-f(y)| \leq\left|f(x)-f_{n}(x)\right|+\left|f_{n}(x)-f_{n}\left(x_{n}\right)\right|+\left|f_{n}\left(x_{n}\right)-f_{n}(y)\right|+\left|f_{n}(y)-f(y)\right| \leq \\
\left\|f-f_{n}\right\|+\left|f_{n}(x)-f_{n}\left(x_{n}\right)\right|+M\left|x_{n}-y\right|+\left\|f-f_{n}\right\|
\end{gathered}
$$

for each $y \in[0,1]$. Convergence of $\left(f_{n}\right)$ to $f$ implies equicontinuity of $\left(f_{n}\right)$ at $x$. So, if $n$ tends to infinity, we get that $|f(x)-f(y)| \leq M|x-y|$. This contradicts $x \notin L_{M}(f)$.

First, we want to focus on functions differentiable almost everywhere.

Proposition 3.3. The set of functions differentiable almost everywhere is Haar-1 in $C[0,1]$.

Proof. Let $\mathcal{A}$ denote the set of functions $f \in C[0,1]$ such that $\lambda(L(f))=1$. Note that each function differentiable almost everywhere is in $\mathcal{A}$. Thus, if we show that $\mathcal{A}$ is Haar-1 in $C[0,1]$, the thesis will follow. 
Recall that $L(f)=\bigcup_{M \in \mathbb{N}} L_{M}(f)$ and $L_{M}(f) \subseteq L_{M+1}(f)$. Thus, $\lambda(L(f))=$ $\lim _{M \rightarrow \infty} \lambda\left(L_{M}(f)\right)$. Consequently,

$$
\mathcal{A}=\bigcap_{k \in \mathbb{N}} \bigcup_{M \in \mathbb{N}}\left\{f \in C[0,1]: \lambda\left(L_{M}(f)\right) \geq \frac{k-1}{k}\right\}
$$

and $\mathcal{A}$ is Borel by Lemma 3.2

Observe that $\mathcal{A}-\mathcal{A}$ is meager as a subset of the set of somewhere Lipschitz functions, which is meager by the Banach theorem [4 (see also the proof of Proposition 4.4). Indeed, if $f \in \mathcal{A}-\mathcal{A}$ then there are $g, h \in \mathcal{A}$ such that $f=g-h$. Since $\lambda(L(g))=1=\lambda(L(h))$, there is $x \in \lambda(L(g)) \cap \lambda(L(h))$. Thus, there are $M_{1}, M_{2} \in \mathbb{N}$ such that $|g(x)-g(y)| \leq M_{1}|x-y|$ and $|h(x)-h(y)| \leq M_{2}|x-y|$ for all $y \in[0,1]$. Thus, for each $y \in[0,1]$ we have:

$$
|f(x)-f(y)| \leq|g(x)-g(y)|+|h(x)-h(y)| \leq\left(M_{1}+M_{2}\right)|x-y| .
$$

The thesis follows now from [5. Corollary 16.3], which states that a Borel set $B$ is Haar-1 if and only if $B-B$ is meager.

Actually, this reasoning gives us something more.

Corollary 3.4. The set of functions $f \in C[0,1]$ such that $\lambda(D(f))>\frac{1}{2}$ is Haar-1 in $C[0,1]$.

Proof. Let $\mathcal{A}$ denote the set of functions $f \in C[0,1]$ such that $\lambda(L(f))>\frac{1}{2}$. Similarly as in the proof of Proposition 3.3, one can show that $\mathcal{A}$ is Borel. If we show that $\mathcal{A}$ is Haar- 1 in $C[0,1]$, the thesis will follow.

We will show that $\mathcal{A}-\mathcal{A}$ is a subset of the set of somewhere Lipschitz functions. Indeed, if $f \in \mathcal{A}-\mathcal{A}$ then there are $g, h \in \mathcal{A}$ such that $f=g-h$. Since $\lambda(L(g))>\frac{1}{2}$ and $\lambda(L(h))>\frac{1}{2}$, there is $x \in \lambda(L(g)) \cap \lambda(L(h))$. Proceeding in the same way as in the proof of Proposition 3.3. we can conclude that $f$ is Lipschitz in $x$.

From now on the proof is entirely the same as the proof of Proposition 3.3 .

Now, we will study functions $f \in C[0,1]$ such that $\lambda(D(f)) \in(0,1)$.

Proposition 3.5. Let $\mathcal{I}$ be a $\sigma$-ideal on $[0,1]$ containing no interval. The set of functions $f \in C[0,1]$ such that neither $D(f)$ nor $[0,1] \backslash D(f)$ belongs to $\mathcal{I}$ is not Haar-finite in $C[0,1]$.

Proof. Denote by $\mathcal{A}$ the set of functions $f \in C[0,1]$ such that $D(f) \notin \mathcal{I}$ and $[0,1] \backslash D(f) \notin \mathcal{I}$.

Let $\varphi:\{0,1\}^{\mathbb{N}} \rightarrow C[0,1]$ be a continuous map. We need to show that $\varphi^{-1}[\mathcal{A}+h]$ is infinite for some $h \in C[0,1]$.

If $\varphi\left[\{0,1\}^{\mathbb{N}}\right]$ is finite, then let $g$ be any element of $\mathcal{A}$ and $h \in C[0,1]$ be such that $\varphi^{-1}[\{h\}]$ is infinite. Observe that $h \in \mathcal{A}+h-g$. Hence, $\varphi^{-1}[\mathcal{A}+h-g]$ is infinite as well.

Assume now that $\varphi\left[\{0,1\}^{\mathbb{N}}\right]$ is infinite and take any injective convergent sequence $\left(f_{n}\right) \in \varphi\left[\{0,1\}^{\mathbb{N}}\right]$. Denote $f=\lim _{n} f_{n}$. For each $n$, let $a_{n}, b_{n}, c_{n} \in\left(\frac{1}{n+2}, \frac{1}{n+1}\right)$ be such that $a_{n}<b_{n}<c_{n}$ and denote $I_{n}^{1}=\left[a_{n}, b_{n}\right]$ and $I_{n}^{2}=\left[b_{n}, c_{n}\right]$. For all $n$ let also $J_{n}=\left[c_{n+1}, a_{n}\right]$ and $g_{n}: J_{n} \rightarrow \mathbb{R}$ be the linear function given by $g_{n}\left(c_{n+1}\right)=0$ and $g_{n}\left(a_{n}\right)=\left(f_{n}-f_{n+1}\right)\left(a_{n}\right)$. Fix any nowhere differentiable function $z \in C[0,1]$ such that $\|z\| \leq 1$ and $z\left(b_{n}\right)=z\left(c_{n}\right)=0$ for each $n$. Such function exists as given any nowhere differentiable function $\tilde{z} \in C[0,1]$ with $z(0)=z(1)=0$ (for instance, the Takagi function) we can define $z \in C[0,1]$ in such a way that 
$z \uparrow\left[b_{n}, c_{n}\right]=\frac{\tilde{z} \circ r_{n}}{\|z\|}$ and $z \uparrow\left[c_{n+1}, b_{n}\right]=\frac{\tilde{z} \circ s_{n}}{\|z\|}$ for each $n$, where $r_{n}:\left[b_{n}, c_{n}\right] \rightarrow[0,1]$ and $s_{n}:\left[c_{n+1}, b_{n}\right] \rightarrow[0,1]$ are linear bijections. Define $h:[0,1] \rightarrow \mathbb{R}$ by:

- $h \uparrow\left[c_{1}, 1\right]=f_{1}\left(c_{1}\right)$

- $h \uparrow I_{n}^{1}=f_{n} \uparrow I_{n}^{1}$ for each $n \in \mathbb{N}$;

- $h \uparrow I_{n}^{2}=f_{n}-\frac{z}{n} \uparrow I_{n}^{2}$ for each $n \in \mathbb{N}$;

- $h\left\lceil J_{n}=\left(f_{n+1}+g_{n}\right) \uparrow J_{n}\right.$ for each $n \in \mathbb{N}$;

- $h(0)=f(0)$.

Note that the function $h$ is continuous (continuity in 0 follows from $\lim _{n} f_{n}=f$, $\lim _{n} \sup _{x \in J_{n}} g_{n}(x)=0$, and $\lim _{n} \frac{\|z\|}{n}=0$ ) and $f_{n} \in \mathcal{A}+h$ for each $n \in \mathbb{N}$ (as $\left(f_{n}-h\right)\left\lceil I_{n}^{1}\right.$ is constant and $\left(f_{n}-h\right)\left\lceil I_{n}^{2}\right.$ is nowhere differentiable). Since $\left(f_{n}\right)$ is injective, we conclude that $\varphi^{-1}[\mathcal{A}+h]$ is infinite.

The next Corollary is immediate.

Corollary 3.6. The set of functions $f \in C[0,1]$ such that $\lambda(D(f)) \in(0,1)$ is not Haar-finite in $C[0,1]$.

Now, we will show that the set of functions $f \in C[0,1]$ such that $D(f)$ is of positive Lebesgue's measure is Haar-countable. The next lemma is a straightforward modification of one of the proofs showing that the Weierstrass function is nowhere Lipschitz.

Lemma 3.7. There is an $a \in(0,1)$ and there is $a b \in \mathbb{N}$ such that for any $x \in(0,1]$ and any increasing sequence $\left(s_{j}\right)$ of positive integers, and any $\left(r_{j}\right) \in\{-1,1\}^{\mathbb{N}}$, the expression

$$
\left|\frac{\sum_{j=0}^{\infty} r_{j} a^{s_{j}}\left(\cos \left(b^{s_{j}} \pi y\right)-\cos \left(b^{s_{j}} \pi x\right)\right)}{y-x}\right|,
$$

where $y$ runs over $[0,1]$, is unbounded.

Proof. Let $a \in(0,1)$ and $b \in \mathbb{N}$ be such that $b$ is odd, $a b>1$, and $\frac{2}{3}-\frac{4 a}{1-a}-\frac{\pi}{a b-1}>0$ (for instance, any $a<\frac{1}{13}$ and any odd $b \in \mathbb{N}$ with $b>\frac{3 \pi+1}{a}$ are good as in this case we have $\frac{4 a}{1-a}<\frac{1}{3}$ and $\left.\frac{\pi}{a b-1}<\frac{1}{3}\right)$.

For each $m \in \mathbb{N}$, let $w_{m} \in \mathbb{Z}$ be such that $x_{m}=x b^{s_{m}}-w_{m} \in\left(-\frac{1}{2}, \frac{1}{2}\right]$ and define $y_{m}=\left(w_{m}-1\right) / b^{s_{m}}$. As $-\frac{1}{2}<x b^{s_{m}}-w_{m} \leq \frac{1}{2}$, we get $x b^{s_{m}}-\frac{3}{2} \leq w_{m}-1<x b^{s_{m}}-\frac{1}{2}$ and $x-\frac{3}{2 b^{s} m} \leq y_{m}<x-\frac{1}{2 b^{s} m}$. Thus, $y_{m} \in[0,1]$ for sufficiently large $m$ (as $x>0$ ).

Fix any $m \in \mathbb{N}$. Since $|\sin (z) / z| \leq 1$, using the formula for the difference of cosines, for each $z$ we have:

$$
\begin{gathered}
\left|\frac{\sum_{j=0}^{m-1} r_{j} a^{s_{j}}\left(\cos \left(b^{s_{j}} \pi y_{m}\right)-\cos \left(b^{s_{j}} \pi x\right)\right)}{y_{m}-x}\right|= \\
=\left|\sum_{j=0}^{m-1} r_{j}(a b)^{s_{j}} \pi \sin \left(\frac{b^{s_{j}} \pi\left(y_{m}+x\right)}{2}\right) \frac{\sin \left(\frac{b^{s_{j}} \pi\left(y_{m}-x\right)}{2}\right)}{\frac{b^{s_{j}} \pi\left(y_{m}-x\right)}{2}}\right| \leq \\
\leq \pi \sum_{j=0}^{s_{m}-1}(a b)^{j}=\frac{\pi\left((a b)^{s_{m}}-1\right)}{a b-1} \leq \frac{\pi(a b)^{s_{m}}}{a b-1} .
\end{gathered}
$$

We will need two observations: if $j \geq m$, then $\cos \left(b^{s_{j}} \pi y_{m}\right)=\cos \left(b^{s_{j}-s_{m}} \pi\left(w_{m}-\right.\right.$ $1))=(-1)^{w_{m}-1}=-(-1)^{w_{m}}\left(\right.$ as $b$ is odd and $\left.w_{m} \in \mathbb{Z}\right)$ and $\cos \left(b^{s_{j}} \pi x\right)=\cos \left(b^{s_{j}-s_{m}} \pi w_{m}\right) \cos \left(b^{s_{j}-s_{m}} \pi x_{m}\right)-\sin \left(b^{s_{j}-s_{m}} \pi w_{m}\right) \sin \left(b^{s_{j}-s_{m}} \pi x_{m}\right)=$ 
DIFFERENTIABILITY OF CONTINUOUS FUNCTIONS IN TERMS OF HAAR-SMALLNESS11

$$
(-1)^{w_{m}} \cos \left(b^{s_{j}-s_{m}} \pi x_{m}\right)
$$

(as $x=\left(x_{m}+w_{m}\right) / b^{s_{m}}$ and $\sin \left(b^{s_{j}-s_{m}} \pi w_{m}\right)=0$ by $\left.b, w_{m} \in \mathbb{Z}\right)$.

Thus, as $b^{s_{m}}\left(x-y_{m}\right)=1+x_{m}$ and $m$ is fixed, we have:

$$
\begin{gathered}
\left|\frac{\sum_{j=m}^{\infty} r_{j} a^{s_{j}}\left(\cos \left(b^{s_{j}} \pi y_{m}\right)-\cos \left(b^{s_{j}} \pi x\right)\right)}{y_{m}-x}\right|= \\
=\left|(a b)^{s_{m}} \frac{\sum_{j=m}^{\infty} r_{j} a^{s_{j}-s_{m}}(-1)(-1)^{w_{m}}\left(1+\cos \left(b^{s_{j}-s_{m}} \pi x_{m}\right)\right)}{-\left(1+x_{m}\right)}\right|= \\
=\left|(a b)^{s_{m}} \sum_{j=m}^{\infty} r_{j} a^{s_{j}-s_{m}} \frac{1+\cos \left(b^{s_{j}-s_{m}} \pi x_{m}\right)}{1+x_{m}}\right| .
\end{gathered}
$$

Recall that $x_{m} \in\left(-\frac{1}{2}, \frac{1}{2}\right]$, which implies $\cos \left(\pi x_{m}\right) \geq 0$. Hence, we can bound the above from below by:

$$
\begin{gathered}
(a b)^{s_{m}}\left(\frac{1}{1+\frac{1}{2}}-\sum_{j=m+1}^{\infty} a^{s_{j}-s_{m}} \frac{1+\cos \left(b^{s_{j}-s_{m}} \pi x_{m}\right)}{1+x_{m}}\right) \geq \\
\geq(a b)^{s_{m}}\left(\frac{2}{3}-4 \sum_{j=1}^{\infty} a^{j}\right)=(a b)^{s_{m}}\left(\frac{2}{3}-\frac{4 a}{1-a}\right) .
\end{gathered}
$$

Therefore, we get:

$$
\begin{aligned}
& \left|\frac{\sum_{j=0}^{\infty} r_{j} a^{s_{j}}\left(\cos \left(b^{s_{j}} \pi y_{m}\right)-\cos \left(b^{s_{j}} \pi x\right)\right)}{y_{m}-x}\right| \geq \\
\geq & || \frac{\sum_{j=m}^{\infty} r_{j} a^{s_{j}}\left(\cos \left(b^{s_{j}} \pi y_{m}\right)-\cos \left(b^{s_{j}} \pi x\right)\right)}{y_{m}-x} \mid- \\
- & \left|\frac{\sum_{j=0}^{m-1} r_{j} a^{s_{j}}\left(\cos \left(b^{s_{j}} \pi y_{m}\right)-\cos \left(b^{s_{j}} \pi x\right)\right)}{y_{m}-x}\right| \geq \\
& \geq(a b)^{s_{m}}\left(\frac{2}{3}-\frac{4 a}{1-a}-\frac{\pi}{a b-1}\right) \stackrel{m \rightarrow \infty}{\longrightarrow} \infty,
\end{aligned}
$$

since $a b>1$ and $\frac{2}{3}-\frac{4 a}{1-a}-\frac{\pi}{a b-1}>0$.

Recall that the $\sigma$-ideal of Lebesgue's null sets is ccc, i.e., every family of pairwise disjoint Borel sets of positive Lebesgue's measure is countable.

Proposition 3.8. The set of functions $f \in C[0,1]$ such that $\lambda(D(f))>0$ is Haarcountable in $C[0,1]$.

Proof. Denote by $\mathcal{A}$ the set of functions $f \in C[0,1]$ such that $\lambda(L(f))>0$ and note that each function $f \in C[0,1]$ such that $\lambda(D(f))>0$ is in $\mathcal{A}$. Moreover, analogously to the proof of Proposition 3.3, we get that $\mathcal{A}$ is Borel, because $\lambda(L(f))=\lim _{M \rightarrow \infty} \lambda\left(L_{M}(f)\right)$ and

$$
\mathcal{A}=\bigcup_{k \in \mathbb{N}} \bigcup_{M \in \mathbb{N}}\left\{f \in C[0,1]: \lambda\left(L_{M}(f)\right) \geq \frac{1}{k}\right\} .
$$


Let $a \in(0,1)$ and $b \in \mathbb{N}$ be as in Lemma 3.7 and let $\varphi:\{0,1\}^{\mathbb{N}} \rightarrow C[0,1]$ be given by:

$$
\varphi(\alpha)(x)=\sum_{j=0}^{\infty}(-1)^{\alpha(j)} a^{j} \cos \left(b^{j} \pi x\right)
$$

for each $\alpha \in\{0,1\}^{\mathbb{N}}$ and $x \in[0,1]$. Continuity of $\varphi$ is obvious as $\lim _{m} \sum_{j=m}^{\infty} a_{j}=0$ and, consequently,

$$
\lim _{m}\left(\sum_{j=m}^{\infty}(-1)^{\alpha(j)} a^{j} \cos \left(b^{j} \pi x\right)\right)=0 .
$$

Continuity of each $\varphi(\alpha)$ follows from Weierstrass M-test and uniform limit theorem.

We claim that $\varphi$ witnesses that $\mathcal{A}$ is Haar-countable. Suppose, to the contrary, that there is an $h \in C[0,1]$ and an uncountable set $T \subseteq\{0,1\}^{\mathbb{N}}$ such that $\varphi(\alpha) \in$ $\mathcal{A}+h$ for each $\alpha \in T$. Without loss of generality, we may assume that $\{i \in \mathbb{N}$ : $\alpha(i) \neq \beta(i)\}$ is infinite for each two distinct $\alpha, \beta \in T$ (since for every $\alpha \in\{0,1\}^{\mathbb{N}}$ there are only countably many $\beta \in\{0,1\}^{\mathbb{N}}$ such that $\{i \in \mathbb{N}: \alpha(i) \neq \beta(i)\}$ is finite).

For $\alpha \in T$, consider the sets $L(\varphi(\alpha)-h) \backslash\{0\}$. They are Borel (cf. Lemma 3.1) and of positive Lebesgue's measure. By the ccc property, we conclude that there are $\alpha, \beta \in T, \alpha \neq \beta$ such that $L(\varphi(\alpha)-h) \cap L(\varphi(\beta)-h) \backslash\{0\} \neq \varnothing$. Let $x$ belong to that set (note that $x \in(0,1])$.

Let $\left(s_{i}\right)$ be the increasing enumeration of the set $\{i \in \mathbb{N}: \alpha(i) \neq \beta(i)\}$ and denote $r_{j}=\beta\left(s_{j}\right)-\alpha\left(s_{j}\right) \in\{-1,1\}$. Observe that:

$$
\frac{\varphi(\alpha)(y)-\varphi(\beta)(y)-\varphi(\alpha)(x)-\varphi(\beta)(x)}{y-x}=2 \frac{\sum_{j=0}^{\infty} r_{j} a^{s_{j}}\left(\cos \left(b^{s_{j}} \pi y\right)-\cos \left(b^{s_{j}} \pi x\right)\right)}{y-x}
$$

for any $y \in[0,1]$. Therefore, by Lemma 3.7 the above expression is unbounded. On the other hand, there are $M_{\alpha}, M_{\beta} \in \mathbb{N}$ witnessing that $\varphi(\alpha)-h$ and $\varphi(\beta)-h$ are Lipschitz at $x$. Thus:

$$
\begin{gathered}
\left|\frac{\varphi(\alpha)(y)-\varphi(\beta)(y)-\varphi(\alpha)(x)-\varphi(\beta)(x)}{y-x}\right| \leq\left|\frac{\varphi(\alpha)(y)-h(y)-\varphi(\alpha)(x)-h(x)}{y-x}\right|+ \\
+\left|\frac{\varphi(\beta)(y)-h(y)-\varphi(\beta)(x)-h(x)}{y-x}\right| \leq M_{\alpha}+M_{\beta}
\end{gathered}
$$

for any $y \in[0,1]$. This is a contradiction.

The next table summarizes results of Sections 2 and 3.

\begin{tabular}{c|c|c|c}
$A=$ & $\{0\}$ & $(0,1)$ & $\{1\}$ \\
\hline$\{f \in \mathcal{S D}: \lambda(D(f)) \in A\} \in$ & Haar-E & Haar-countable & Haar-1 \\
\hline$\{f \in \mathcal{S D}: \lambda(D(f)) \in A\} \notin$ & Haar-countable & Haar-finite & -
\end{tabular}

\section{Multidimensional Case}

In this Section, we study nowhere differentiable functions on $[0,1]^{k}$, i.e., functions defined on $[0,1]^{k}$ which do not have a finite directional derivative at any point along any vector (at points from the boundary of $[0,1]^{k}$ we require that there is no finite directional one-sided derivative along any vector). Such functions exist (actually, in Proposition 4.4 we even show that the set of such functions is comeager in $C[0,1]^{k}$ ), however, it is hard to find a suitable example in the literature. Therefore, below we provide one for $k=2$ (with an informal proof). 
Example 4.1. Let $T \in C[0,1]$ be the Takagi function, i.e.,

$$
T(x)=\sum_{n=0}^{+\infty} \frac{1}{2^{n}} \operatorname{dist}\left(2^{n} x, \mathbb{Z}\right) .
$$

We define a new function on $[0,1]^{2}$ :

$$
F\left(x_{1}, x_{2}\right)=\tilde{T}\left(\sqrt{x_{1}^{2}+x_{2}^{2}}\right)
$$

(here $\tilde{T}(x)=T(\operatorname{frac}(x))$, where frac $(x)$ is the fractional part of $x)$. Choose a point $(0,0) \neq\left(\chi_{0}, \chi_{1}\right) \in[0,1]^{2}$ and a unit vector $v \in \mathbb{R}^{2}$. Denote by $\ell$ a line that goes through $\left(\chi_{1}, \chi_{2}\right)$ and that is parallel to $v$. For every point $\left(x_{1}, x_{2}\right)$ of $\ell \cap[0,1]^{2}$, consider the circle $\mathcal{O}_{\left(x_{1}, x_{2}\right)}$ centered at the origin that passes through $\left(x_{1}, x_{2}\right)$. Let $x$ be the $x$-intercept of $\mathcal{O}_{\left(x_{1}, x_{2}\right)}$ and define a one-to-one correspondence between $\left[\inf \left\{\sqrt{x_{1}^{2}+x_{2}^{2}}:\left(x_{1}, x_{2}\right) \in \ell \cap[0,1]^{2}\right\}, \sup \left\{\sqrt{x_{1}^{2}+x_{2}^{2}}:\left(x_{1}, x_{2}\right) \in \ell \cap[0,1]^{2}\right\}\right]$ and $\ell \cap[0,1]^{2}$ by $x \mapsto\left(x_{1}, x_{2}\right)$. We will always denote by $x$ a point on $[0,1]$ that corresponds to the point $\left(x_{1}, x_{2}\right)$ on $\ell$. Note that $F\left(x_{1}, x_{2}\right)=F(x, 0)=\tilde{T}(x)$. Denote by $d_{e}^{2}$ the standard euclidean metric on $[0,1]^{2}$. Moreover, denote by a and $b$ the slope of $\ell$ and its $y$-intercept respectively. Choose two sequences $\left(u^{n}\right)$ and $\left(v^{n}\right)$ that tend to $\chi$ (recall that $\chi$ is the $x$-intercept of $\left.\mathcal{O}_{\left(\chi_{1}, \chi_{2}\right)}\right)$. Now, for every $n$, assume that all elements of $\left(u^{k}\right)_{k \geq n}$ and $\left(v^{k}\right)_{k \geq n}$ are within some interval $\left(\alpha_{n}, \beta_{n}\right)$. Without loss of generality, we may assume that both $\left(\alpha_{n}\right)$ and $\left(\beta_{n}\right)$ tend to $\chi$. Using elementary analytical geometry and a little bit of estimation, we can show that:

$\frac{\alpha_{n} \sqrt{a^{2}+1}}{\sqrt{\beta_{n}^{2}\left(a^{2}+1\right)-b^{2}}}\left|u^{n}-v^{n}\right| \leq d_{e}^{2}\left(\left(u_{1}^{n}, u_{2}^{n}\right),\left(v_{1}^{n}, v_{2}^{n}\right)\right) \leq \frac{\beta_{n} \sqrt{a^{2}+1}}{\sqrt{\alpha_{n}^{2}\left(a^{2}+1\right)-b^{2}}}\left|u^{n}-v^{n}\right|$ (i.e., the euclidean metric on $\left(\alpha_{n}, \beta_{n}\right)$ is equivalent to $d_{e}^{2}\left\lceil\left\{x \in \ell: d_{e}^{2}((0,0), x) \in\right.\right.$ $\left.\left.\left(\alpha_{n}, \beta_{n}\right)\right\}^{2}\right)$. For simplicity, let $c_{n}=\frac{\alpha_{n} \sqrt{a^{2}+1}}{\sqrt{\beta_{n}^{2}\left(a^{2}+1\right)-b^{2}}}$ and $d_{n}=\frac{\beta_{n} \sqrt{a^{2}+1}}{\sqrt{\alpha_{n}^{2}\left(a^{2}+1\right)-b^{2}}}$. Observe that $\left(c_{n}\right)$ and $\left(d_{n}\right)$ have the same limit. Note that:

$$
c_{n} \frac{\left|F\left(u_{1}^{n}, u_{2}^{n}\right)-F\left(v_{1}^{n}, v_{2}^{n}\right)\right|}{d_{e}^{2}\left(\left(u_{1}^{n}, u_{2}^{n}\right),\left(u_{1}^{n}, u_{2}^{n}\right)\right)} \leq \frac{\left|\tilde{T}\left(u^{n}\right)-\tilde{T}\left(v^{n}\right)\right|}{\left|u^{n}-v^{n}\right|} \leq d_{n} \frac{\left|F\left(u_{1}^{n}, u_{2}^{n}\right)-F\left(v_{1}^{n}, v_{2}^{n}\right)\right|}{d_{e}^{2}\left(\left(u_{1}^{n}, u_{2}^{n}\right),\left(u_{1}^{n}, u_{2}^{n}\right)\right)} .
$$

Finally, it follows by the Squeeze Theorem that if $F$ is differentiable at $\left(\chi_{1}, \chi_{2}\right)$ in the direction $v$, then $T$ must be differentiable at $\chi$. This is impossible. Thus, $F$ is not differentiable at any point of $[0,1]^{2} \backslash\{(0,0)\}$ in any direction. To complete the proof, proceed analogously for $\left(\chi_{0}, \chi_{1}\right)=(0,0)$ and use the fact that $T$ does not possess a finite one-sided derivative at 0.

Now, we want to show that, unlike the one-dimensional case, the set of somewhere differentiable functions on $[0,1]^{k}$ is not Haar-null. Actually, this follows from a more general fact. We will need the following notion.

A subset $A$ of an abelian Polish group $X$ is called thick if for any compact set $K \subseteq X$ there is an $x \in X$ such that $K+x \subseteq A$ (for more on thick sets see [5, Section 7]).

Remark 4.2. The following are equivalent for any Borel set A:

(a) $A$ is thick;

(b) $A$ is not Haar- $(\mathcal{P}(X) \backslash\{X\})$;

(c) $A$ is not Haar-I for any proper semi-ideal $\mathcal{I}$. 
Moreover, if $A$ is arbitrary, then (b) and (c) are equivalent and (a) implies both of them.

Proof. Indeed, $(\mathrm{b}) \Longleftrightarrow(\mathrm{c})$ is trivial. As $f\left[\{0,1\}^{\mathbb{N}}\right]$ is compact for every continuous $f$, thickness of $A$ ensures us that $A$ is not Haar- $(\mathcal{P}(X) \backslash\{X\})$. Conversely, if $A$ is not thick, then the compact set $K$ witnessing it is a continuous image of $\{0,1\}^{\mathbb{N}}$. This continuous map witnesses that $A$ is $\operatorname{Haar}-(\mathcal{P}(X) \backslash\{X\})$ provided that it is Borel.

We are ready to prove the aforementioned general result.

Proposition 4.3. Let $k>1$. The set of somewhere differentiable functions is thick in $C[0,1]^{k}$.

Proof. Firstly, we will assume that $k=2$.

Let $C$ be the ternary Cantor set (which is homeomorphic to $\{0,1\}^{\mathbb{N}}$ ) and $\varphi: C \rightarrow$ $C[0,1]^{2}$ be continuous. We will define a continuous function $f:[0,1]^{2} \rightarrow \mathbb{R}$ such that for all $c \in C$ the function $\varphi(c)-f$ is differentiable along $(0,1)$ in each point of the form $(c, x), x \in[0,1]$.

Let $F: C \times[0,1] \rightarrow \mathbb{R}$ be given by $F\lceil\{c\} \times[0,1]=\varphi(c)\lceil\{c\} \times[0,1]$ for each $c \in C$.

We need to show that $F$ is continuous. Fix any $\left(c_{0}, y_{0}\right) \in C \times[0,1]$ and $\varepsilon>0$. Since $\varphi\left(c_{0}\right)$ is continuous at $\left(c_{0}, y_{0}\right)$, there is an open neighborhood $\left(c_{0}, y_{0}\right) \in U \subseteq$ $[0,1]^{2}$ such that $\left|\varphi\left(c_{0}\right)(x, y)-\varphi\left(c_{0}\right)\left(c_{0}, y_{0}\right)\right|<\frac{\varepsilon}{2}$ for every $(x, y) \in U$. Moreover, since $\varphi$ is continuous, there is an open neighborhood $c_{0} \in V \subseteq[0,1]$ such that $\left\|\varphi(c)-\varphi\left(c_{0}\right)\right\|<\frac{\varepsilon}{2}$ for each $c \in V \cap C$. Thus, we have:

$$
\begin{gathered}
\left|F(c, y)-F\left(c_{0}, y_{0}\right)\right|=\left|\varphi(c)(c, y)-\varphi\left(c_{0}\right)\left(c_{0}, y_{0}\right)\right| \leq \\
\leq\left|\varphi(c)(c, y)-\varphi\left(c_{0}\right)(c, y)\right|+\left|\varphi\left(c_{0}\right)(c, y)-\varphi\left(c_{0}\right)\left(c_{0}, y_{0}\right)\right| \leq \\
\leq|| \varphi(c)-\varphi\left(c_{0}\right) \|+\left|\varphi\left(c_{0}\right)(c, y)-\varphi\left(c_{0}\right)\left(c_{0}, y_{0}\right)\right|<\frac{\varepsilon}{2}+\frac{\varepsilon}{2}=\varepsilon
\end{gathered}
$$

for all $(c, y) \in U \cap((V \cap C) \times[0,1])$. Hence, $F$ is continuous.

Using Tietze extension theorem we get a continuous function $f:[0,1]^{2} \rightarrow \mathbb{R}$ such that $f\lceil C \times[0,1]=F$. Then, $\varphi(c)-f\lceil\{c\} \times[0,1]$ is constantly equal to 0 for each $c \in C$. Hence, $\varphi(c)-f$ is somewhere differentiable and we are done.

If $k>2$ then define $F: C \times[0,1] \times\{0\}^{k-2} \rightarrow \mathbb{R}$ by $F\left\lceil\{c\} \times[0,1] \times\{0\}^{k-2}=\right.$ $\varphi(c)\left\lceil\{c\} \times[0,1] \times\{0\}^{k-2}\right.$ for each $c \in C$ and observe that $F$ is continuous for the same reason as above. Thus, using Tietze extension theorem we get a continuous function $f:[0,1]^{k} \rightarrow \mathbb{R}$ such that $\varphi(c)-f$ is somewhere differentiable.

Although the set of somewhere differentiable functions on $[0,1]^{k}$ is not Haar- $\mathcal{I}$ for any semi-ideal $\mathcal{I}$, it is meager. In the one-dimensional case this was shown by Banach (see [4). The authors find it surprising (and remain skeptical) that no multidimensional case of this theorem can be found in the literature, as the following reasoning is only a slight modification of the one-dimensional proof.

Proposition 4.4. The set of nowhere differentiable functions on $[0,1]^{k}$ is comeager in $C[0,1]^{k}$.

Proof. We will consider $k=2$, but the argument works for other $k$ 's as well. Let $E_{n}^{0,0}$ be the set of these functions $f \in C[0,1]^{2}$ such that there is a pair $(x, y) \in$ $\left[0,1-\frac{1}{n+1}\right]^{2}$ and there is a unit vector $\left(v_{1}, v_{2}\right) \in\left[\frac{1}{n+1}, 1\right] \times[-1,1]$ such that for all 
DIFFERENTIABILITY OF CONTINUOUS FUNCTIONS IN TERMS OF HAAR-SMALLNESS15

$h \in\left(0, \frac{1}{n}\right)$ it holds that $\left|f\left((x, y)+h\left(v_{1}, v_{2}\right)\right)-f(x, y)\right|<n h$. Define $E_{n}^{0,1}, E_{n}^{1,0}$ and $E_{n}^{1,1}$ analogously replacing $R_{n}^{0,0}=\left[0,1-\frac{1}{n+1}\right]^{2}$ with $R_{n}^{0,1}=\left[0,1-\frac{1}{n+1}\right] \times\left[\frac{1}{n+1}, 1\right]$, $R_{n}^{1,0}=\left[\frac{1}{n+1}, 1\right] \times\left[0,1-\frac{1}{n+1}\right]$ and $R_{n}^{1,1}=\left[\frac{1}{n+1}, 1\right]^{2}$, respectively. Similarly, let $F_{n}^{i, j}$ be the set of these functions $f \in C[0,1]^{2}$ such that there is a pair $(x, y) \in R_{n}^{i, j}$ such that for all $h \in\left(0, \frac{1}{n}\right)$ it holds that $|f(x, y+h)-f(x, y)|<n h$. It suffices to prove that for each $i, j \in\{0,1\}$ and each $n \in \mathbb{N}$ :

a) $E_{n}^{i, j}$ is closed;

b) $F_{n}^{i, j}$ is closed;

c) $E_{n, j}^{i, j}$ is nowhere dense;

d) $F_{n}^{i, j}$ is nowhere dense;

and also that:

e) $C[0,1]^{2} \backslash \bigcup_{n \in \mathbb{N}} \bigcup_{i, j \in\{0,1\}}\left(E_{n}^{i, j} \cup F_{n}^{i, j}\right)$ is a subset of the set of nowhere differentiable functions on $[0,1]^{2}$.

For $a$ ) and $b$ ), it is enough to use the argument from the standard one-dimensional case. We will show that $E_{m}^{0,0}$ is closed. The other cases are similar.

Let $\left(f_{n}\right) \subseteq E_{m}^{0,0}$ and denote $f=\lim _{n} f_{n}$. For each $n \in \mathbb{N}$ there is $\left(x_{n}, y_{n}\right) \in$ $\left[0,1-\frac{1}{m+1}\right]^{2}$ and there is a unit vector $\left(v_{1}^{n}, v_{2}^{n}\right) \in\left[\frac{1}{m+1}, 1\right] \times[-1,1]$ such that for all $h \in\left(0, \frac{1}{m}\right)$ it holds that $\left|f_{n}\left(\left(x_{n}, y_{n}\right)+h\left(v_{1}^{n}, v_{2}^{n}\right)\right)-f_{n}\left(x_{n}, y_{n}\right)\right|<m h$. By the Bolzano-Weierstrass theorem, passing to a subsequence, if necessary, without loss of generality we can assume that $\lim _{n}\left(x_{n}, y_{n}\right)=(x, y) \in\left[0,1-\frac{1}{m+1}\right]^{2}$ and $\lim _{n}\left(v_{1}^{n}, v_{2}^{n}\right)=\left(v_{1}, v_{2}\right) \in\left[\frac{1}{m+1}, 1\right] \times[-1,1]$. Fix $h \in\left(0, \frac{1}{m}\right)$ and observe that:

$$
\begin{gathered}
\left|f\left((x, y)+h\left(v_{1}, v_{2}\right)\right)-f(x, y)\right| \leq\left|f\left((x, y)+h\left(v_{1}, v_{2}\right)\right)-f\left(\left(x_{n}, y_{n}\right)+h\left(v_{1}^{n}, v_{2}^{n}\right)\right)\right|+ \\
+\left|f\left(\left(x_{n}, y_{n}\right)+h\left(v_{1}^{n}, v_{2}^{n}\right)\right)-f_{n}\left(\left(x_{n}, y_{n}\right)+h\left(v_{1}^{n}, v_{2}^{n}\right)\right)\right|+ \\
+\left|f_{n}\left(\left(x_{n}, y_{n}\right)+h\left(v_{1}^{n}, v_{2}^{n}\right)\right)-f_{n}\left(\left(x_{n}, y_{n}\right)\right)\right|+\left|f_{n}\left(\left(x_{n}, y_{n}\right)\right)-f\left(\left(x_{n}, y_{n}\right)\right)\right|+ \\
+\left|f\left(\left(x_{n}, y_{n}\right)\right)-f((x, y))\right| \leq\left|f\left((x, y)+h\left(v_{1}, v_{2}\right)\right)-f\left(\left(x_{n}, y_{n}\right)+h\left(v_{1}^{n}, v_{2}^{n}\right)\right)\right|+ \\
+\left\|f-f_{n}\right\|+m h+\left\|f-f_{n}\right\|+\left|f\left(\left(x_{n}, y_{n}\right)\right)-f((x, y))\right| .
\end{gathered}
$$

If $n \rightarrow+\infty$ then continuity of $f$ at $(x, y)$ and at $\left((x, y)+h\left(v_{1}, v_{2}\right)\right)$ together with $f=\lim _{n} f_{n}$ gives us that:

$$
\left|f\left((x, y)+h\left(v_{1}, v_{2}\right)\right)-f(x, y)\right| \leq m h
$$

and we can conclude that $f \in E_{m}^{0,0}$.

For $c$ ), we will prove that for every two-dimensional piecewise linear function $g$ and every $\varepsilon>0$ there is a function $f \notin E_{n}^{0,0}$ such that the norm of $f-g$ is below $\varepsilon$ (the cases of $E_{n}^{0,1}, E_{n}^{1,0}$ and $E_{n}^{1,1}$ are analogous). As the set of all two-dimensional piecewise linear functions is dense in $C[0,1]^{2}$, the thesis will follow.

Let $g$ and $\varepsilon$ be defined as above and let $M$ be equal to the maximal slope of $g$. Subsequently, choose $m$ such that $\frac{m}{n+1} \frac{\varepsilon}{2}>M+n$. Now, define a function $f(x, y)=g(x, y)+\frac{\varepsilon}{2} \operatorname{dist}(m x, \mathbb{Z})$. It is easy to see that $|f(x, y)-g(x, y)|<\varepsilon$ for all $(x, y)$. Let $(x, y) \in\left[0,1-\frac{1}{n+1}\right]^{2}$ and let $v=\left(v_{1}, v_{2}\right) \in\left[\frac{1}{n+1}, 1\right] \times[-1,1]$ be a unit vector. Observe that:

$$
\left|\frac{\partial f}{\partial v}\right|=\left|\frac{\partial g}{\partial v}+\frac{\varepsilon}{2} m v_{1} \frac{\partial \operatorname{dist}(\cdot, \mathbb{Z})}{\partial x}\right|>n
$$

as $\left|\frac{\partial \operatorname{dist}(\cdot, \mathbb{Z})}{\partial x}\right|=1$ and $v_{1}>\frac{1}{n+1}$. 
For $d$ ), we just repeat an argument we just gave: we choose $m$ such that $m \frac{\varepsilon}{2}>$ $M+n$, define $f(x, y)=g(x, y)+\frac{\varepsilon}{2} \operatorname{dist}(m y, \mathbb{Z})$ and observe that:

$$
\left|\frac{\partial f}{\partial y}\right|=\left|\frac{\partial g}{\partial y}+\frac{\varepsilon}{2} m \frac{\partial \operatorname{dist}(\cdot, \mathbb{Z})}{\partial y}\right|>n \text {. }
$$

Finally, for $e$ ), let us suppose that $f \in C[0,1]^{2} \backslash\left(\bigcup_{n \in \mathbb{N}} \bigcup_{i, j \in\{0,1\}} E_{n}^{i, j} \cup \bigcup_{n \in \mathbb{N}} F_{n}^{i, j}\right)$, choose $(x, y) \in(0,1)^{2}$, and choose a unit vector $v$. There are two cases to consider, but they essentially come down to the same argument. If the vector $v$ is different than $(0,1)$, we will use the sets $E_{n}^{i, j}$, if not, we will use $F_{n}^{i, j}$. Without loss of generality, we will continue under assumption that $v=(0,1)$. Let $i, j \in\{0,1\}$ be such that $(x, y) \in \bigcap_{n \in \mathbb{N}} R_{n}^{i, j}$. Since $f \in \bigcap_{n \in \mathbb{N}} C[0,1]^{2} \backslash F_{n}^{i, j}$, it follows that for all $n \in \mathbb{N}$ there is an $h_{n} \in\left(0, \frac{1}{n}\right)$ such that $\left|f\left(x, y+h_{n}\right)-f(x, y)\right| \geq n h_{n}$. It is now easy to see that $f$ is nowhere differentiable.

We end with an open problem which occurred during our studies.

Here, we examine the function $F:[0,1]^{2} \rightarrow \mathbb{R}$ given by $F(x, y)=T(x)+T(y)$, where $T:[0,1] \rightarrow \mathbb{R}$ is the Takagi's function. We considered this function in the context of nowhere differentiable functions on $[0,1]^{2}$ as $F$ seemed to be a nice example of such a function. It is obvious that it is differentiable neither along $(1,0)$ nor along $(0,1)$. Moreover, we managed to obtain one partial result: $F$ is differentiable at no point along $(1,1)$. However, we were unable to solve the following.

Problem 4.5. Is $F$ nowhere differentiable on $[0,1]^{2}$ ?

\section{REFERENCES}

[1] P.C. Allaart, K. Kawamura, The improper infinite derivatives of Takagi's nowhere-differentiable function, J. Math. Anal. Appl. 372 (2010), 656-665.

[2] P.C. Allaart, K. Kawamura, The Takagi function: a survey, Real Anal. Exchange 37 (2011), 1-54.

[3] M. Balcerzak, Can ideals without ccc be interesting?, Top. App. 55 (1994), 251-260.

[4] S. Banach, Über die Baire'sche Kategorie gewisser Funktionenmengen, Studia Math. 3 (1931), 174-179.

[5] T. Banakh, S. Głąb, E. Jabłońska, J. Swaczyna, Haar-I sets: looking at small sets in Polish groups through compact glasses, submitted, available online at: https://arxiv.org/abs/1803.06712 (last accessed July 5th, 2020).

[6] T. Banakh, N. Lyaskovska, D. Repovš, Packing index of subsets in Polish groups, Notre Dame J. Formal Logic 50 (2009), 453-468.

[7] T. Bartoszyński, H. Judah, Set theory: On the structure of the real line, A K Peters, Ltd., Wellesley, MA, 1995.

[8] J.P.R. Christensen, On sets of Haar measure zero in abelian Polish groups, Israel J. Math. 13 (1972), 255-260.

[9] U.B. Darji, On Haar meager sets, Top. Appl. 160 (2013), 2396-2400.

[10] U. B. Darji, T. Keleti, Covering $\mathbb{R}$ with translates of a compact set, Proc. Amer. Math. 131 (2003), 2593-2596.

[11] M. Elekes, J. Steprāns, Less than $2^{\omega}$ many translates of a compact nullsets may cover the real line, Fund. Math. 181 (2004), 89-96. 
DIFFERENTIABILITY OF CONTINUOUS FUNCTIONS IN TERMS OF HAAR-SMALLNESS17

[12] B.R. Hunt, The prevalance of continuous nowhere differentiable functions, Proc. Amer. Math. Soc. 3 (1994), 711-717.

[13] B.R. Hunt, T. Sauer, J.A. Yorke, Prevalence: a translation-invariant "almost every" on infinite-dimensional spaces, Bull. Amer. Math. Soc. 27 (1992), 217238.

[14] A. Kwela, Haar-smallest sets, Top. App. 267 (2019), 106892.

[15] T. Takagi, A simple example of continuous function without derivative, Proc. Phys. Math. Soc. Japan 1 (1903), 176-177.

[16] Z. Zahorski, Sur l'ensemble des points de non-dérivabilité d'une fonction continue, Bulletin de la S. M. F. 74 (1946), 147-178.

[17] P. Zakrzewski, On Borel sets belonging to every invariant $c c c \sigma$-ideal on $2^{\omega}$, Proc. Amer. Math. Soc. 141 (2013), 1055-1065.

Institute of Mathematics, Faculty of Mathematics, Physics and Informatics, UniverSity of Gdańsk, Ul. Wita Stwosza 57, 80-308 Gdańsk, Poland

E-mail address: Adam.Kwela@ug.edu.pl

Institute of Mathematics, Faculty of Mathematics, Physics and Informatics, UniverSity of Gdańsk, ul. Wita Stwosza 57, 80-308 Gdańsk, Poland

E-mail address: woloszyn.w.a@gmail.com 